\title{
Identification of a rice drying model with an improved sequential optimal design of experiments
}

\author{
Daniel Goujot ${ }^{\mathrm{a}, \mathrm{b}, *}$, Xuân Meyer ${ }^{\mathrm{c}}$, Francis Courtois ${ }^{\mathrm{b}, \mathrm{a}}$ \\ a INRA, UMR1145 Ingénierie Procédés Aliments, 1 avenue des Olympiades, F-91300 Massy, France \\ b AgroParisTech, UMR1145 Ingénierie Procédés Aliments, 1 avenue des Olympiades, F-91300 Massy, France \\ ' Université de Toulouse, Laboratoire de Génie Chimique, UMR CNRS/INPT/UPS 5503, BP 34038, 4 allée Emile Monso, 31030 Toulouse cedex 4, France
}

Keywords:

Design of experiments (DoE)

Non-linear system

Drying model

Parameter estimation

Uncertainties

\begin{abstract}
A B S T R A C T
Getting relevant parameter estimation of a non-linear model is often a hard task from both an experimental and numerical point of view. The objective of optimally designed experiments procedure is to diminish the experimental effort needed such that the identification is within acceptable confidence ranges. After each experiment, the next experiment is optimally designed, taking into account all past experimental results. It allows quality information to be extracted from the experimental data with less experimental time and resource consumption.

In this paper, we present an original approach and implementation of the classical A-, D- and Eoptimality on the estimation of 5 unknown (transfer related) coefficients in a compartmental model used to describe the convective drying of rice. The originality of our method is that it uses reparameterization of both parameter and protocol vectors which permits to avoid using a global optimization algorithm. The presented method is implemented in Matlab as a Toolbox and fully tested on a pilot plant. The case study (drying of rice) is typical in the field of process engineering: the dynamic model is strongly non-linear in its parameters and cannot be analytically solved. In addition, the specific technical constrains (inertias, limits, etc.) on the pilot are explicitly taken into account for improved experimental feasibility.

In this drying application, three experiments with non-constant drying conditions are shown to be quite as effective as a two-factor three-level grid of nine experiments at constant conditions, with only one third of the experimental effort.
\end{abstract}

\section{Introduction}

The design of experiment (DoE) can be considered as the optimization of the experimental effort required to identify unknown parameters with minimal confidence intervals.

In this paper, we present an original approach and implementation of the experimental estimation of 5 unknown (transfer related) coefficients with a minimal number of experiments in the field of drying of foodstuffs.

Apart from pure empirical approaches, it is quite common to use freeware or commercial software to calculate a multi factor multi level plan. Implicitly, a simple LP (linear in its parameters) model is assumed. Furthermore, the model is simple, algebraic and

\footnotetext{
* Corresponding author at: INRA, UMR1145 Ingénierie Procédés Aliments, 1 avenue des Olympiades, F-91300 Massy, France. Tel.: +3316993 5188; fax: +33169935185.

E-mail addresses: daniel.goujot@agroparistech.fr, daniel.goujot@gmail.com (D. Goujot), xuan.meyer@ensiacet.fr (X. Meyer), francis.courtois@agroparistech.fr (F. Courtois).
}

usually formulated as a polynomial. This kind of calculation is well documented in many books or websites. Unfortunately, using this design methodology on dynamic models (i.e. described by differential equations) with strong non-linearities in their parameters is just a work around leading to numerous experiments and minimal guarantee on future parameter values.

On the other hand, some recent works [1-3] have described optimal DoE methods for non-LP models (DoENL methods). For instance, the X-optimality criterion was introduced by [2] to deal with such problems. Their design methodology is requiring billions of simulations which is incompatible with most engineering models where no analytical solution exists, hence requiring lengthy numerical integration. This, and the induced complications, can explain why most works dealing with non-LP models are using optimal DoE methods for LP models (DoEL methods). As a matter of fact, in a recent and very interesting review [4], almost all papers listed are using a DoEL method even with non-LP models. In [4], about $70 \%$ of all papers listed are based on an analytical (true or approximate) solution of the non linear model. Unfortunately, in food engineering, having an analytical solution is not that common. 


\section{Nomenclature}

\section{Roman letters}

$a_{w} \quad$ water activity, see Eq. (5)

d.b. dry basis, for moisture content, short hand unit for $g_{w p} g_{d m}^{-1}$

$D \quad$ diffusion coefficient $\left(\mathrm{m}^{2} / \mathrm{s}\right)$

$h$ heat transfer coefficient, see Section 2.3.1 $\left(\mathrm{W} \mathrm{m}^{-2} \mathrm{~K}^{-1}\right)$

$l \quad$ volume to surface ratio $(\mathrm{m})$

$\log _{10}$ decimal logarithm function

$M_{\text {Fisher }}$ Fisher information matrix, $5 \times 5$ square matrix, see (10) Section 2.4

$M_{\text {Jacobian }}$ Jacobian sensibility matrix, see (8) Section 2.4 (d.b.)

$M_{r} \quad$ lack-of-fit vector, see Section 2.5 (d.b.)

$N_{\varphi} \quad$ number of weighting in experiment of protocol $\varphi$

NA place holder for something not available

$p \quad$ parameter

$P \quad$ pressure (Pa)

$t \quad$ time $(\mathrm{s})$

$t_{\varphi, i} \quad$ time of $i$ th weighting in experiment of protocol $\varphi(\mathrm{s})$

$T \quad$ product temperature $\left({ }^{\circ} \mathrm{C}\right)$

$\frac{X}{X} \quad$ product moisture content (d.b.)

$\bar{X} \quad$ mean product moisture content (d.b.)

$Y \quad$ air moisture content $\left(g_{v a} g_{d a}^{-1}\right)$

\section{Greek letters}

$\theta \quad$ vector of parameters shown in Eq. (7)

$\theta_{1}, \theta_{2}, \ldots$ coefficients of the vector of parameters

$\rho \quad$ density $\left(\mathrm{kg} / \mathrm{m}^{3}\right)$

$\sigma_{f}^{2} \quad$ lack of fit (residual) variance, reflecting the lack of prediction of experimental data by model identified with these data (d.b. ${ }^{2}$ )

$\sigma_{v}^{2} \quad$ lack of validation variance, reflecting the lack of prediction of experimental data by model identified independently (d.b. ${ }^{2}$ )

$\Sigma \quad$ variance-covariance matrix, see Section 2.4 (d.b. ${ }^{2}$ )

$\tau \quad$ compartment volume ratio, see Section 2.1

$\varphi \quad$ vector of protocols shown in Eq. (6)

$\chi^{2} \quad$ cumulated normal distribution [36, Section 10.2, p. 144]

\section{Subscripts}

0 at beginning of methodology, or at last set point change

1,2 inner compartment, outer compartment

$3,4,5, \ldots$ relative to constants, see Constants

a air

da dry air

$d m \quad$ dry matter of rice

sat about saturation of vapor in air

$w p \quad$ water in product

$v a, v p \quad$ vapor in air, in product, see Eq. (4)

$\infty \quad$ relative to set point values

$\infty 1, \infty 2, \ldots$ first, second, ... set point values

\section{Superscripts}

$T \quad$ transpose of a matrix

$-1 \quad$ inverse of a matrix

-2 square of inverse of a matrix

$-1 / 2$ square root of inverse

experimentally realized

mean on the rice

\begin{tabular}{|c|c|}
\hline \multicolumn{2}{|c|}{ Constants } \\
\hline$a_{3}$ & $\begin{array}{l}\text { decimal logarithm of saturated vapor pressure at } \\
0^{\circ} \mathrm{C}(2.7858)\end{array}$ \\
\hline$a_{4}$ & $\begin{array}{l}\text { decimal logarithm of maximal saturated vapor pres- } \\
\text { sure }(4.7142)\end{array}$ \\
\hline$c_{\text {colburn }}$ & constant from Colburn analogy $[43]\left(65.0 \mathrm{~m}^{3} \mathrm{KJ}^{-1}\right)$ \\
\hline$c_{p w}$ & water specific capacity $\left(4210 \mathrm{~J} \mathrm{~kg}^{-1} \mathrm{~K}^{-1}\right)$ \\
\hline$c_{p d m}$ & specific capacity of calorific Rice [44] \\
\hline & (http://rpaulsingh.com/teaching/SpecificHeat1.htm \\
\hline & $\begin{array}{l}\text { Arrowhead Mills'basmati), white unprepared } \\
\left.\text { (1653 } \mathrm{J} \mathrm{kg}^{-1} \mathrm{~K}^{-1}\right)\end{array}$ \\
\hline$H_{R a 3}$ & $\begin{array}{l}\text { relative humidity factor of Gompertz inertia } \\
(0.5433 \%)\end{array}$ \\
\hline$H_{R a 4}$ & $\begin{array}{l}\text { relative humidity factor of exponential inertia } \\
(0.8395 \%)\end{array}$ \\
\hline$H_{R a 5}$ & $\begin{array}{l}\text { relative humidity normalization factor of exponen- } \\
\text { tial relative humidity inertia }(0.8596 \%)\end{array}$ \\
\hline$H_{R a 6}$ & $\begin{array}{l}\text { relative humidity normalization factor of Gompertz } \\
\text { relative humidity inertia }(23.311 \%)\end{array}$ \\
\hline$L_{v}$ & latent heat of vaporization $\left(2.357 \times 10^{6} \mathrm{~J} \mathrm{~kg}^{-1}\right)$ \\
\hline$l_{1}$ & length of inner compartment $[45, \mathrm{p} .91, \mathrm{r}](0.5 \mathrm{~mm})$ \\
\hline$S_{\text {spec }}$ & $\begin{array}{l}\text { specific surface to volume ratio, measured with the } \\
\text { spheroid prolate hypothesis }\left(1989 \mathrm{~m}^{-1}\right)\end{array}$ \\
\hline$T_{3}$ & $\begin{array}{l}\text { temperature with water pressure equal to } 10^{\left(a_{3}+a_{4}\right) / 2} \\
\left(237.3^{\circ} \mathrm{C}\right)\end{array}$ \\
\hline$T_{4}$ & $\begin{array}{l}\text { maximal temperature at which water activity of rice } \\
\text { is } 0\left(2.5457^{\circ} \mathrm{C}\right)\end{array}$ \\
\hline$T_{a 3}$ & temperature factor of Gompertz inertia ( $0.9993 \mathrm{~K})$ \\
\hline$T_{a 4}$ & temperature factor of exponential inertia $(0.0369 \mathrm{~K})$ \\
\hline$T_{a 5}$ & $\begin{array}{l}\text { temperature normalization factor of exponential } \\
\text { temperature inertia }(1.5810 \mathrm{~K})\end{array}$ \\
\hline$T_{a 6}$ & $\begin{array}{l}\text { temperature normalization factor of Gompertz tem- } \\
\text { perature inertia }(1.0231 \mathrm{~K})\end{array}$ \\
\hline$T_{\max }$ & maximal temperature of equipment $\left(100^{\circ} \mathrm{C}\right)$ \\
\hline$T_{\min }$ & temperature of far air atmosphere $\left(20^{\circ} \mathrm{C}\right)$ \\
\hline$t_{3}$ & time delay of Gompertz inertia (833.1221 s) \\
\hline$t_{4}$ & characteristic time of Gompertz inertia (394.9341 s) \\
\hline$t_{5}$ & characteristic time of exponential inertia (60s) \\
\hline$t_{6}$ & time interval between two weighting (60s) \\
\hline$t_{\max }$ & duration of each experiment (7200 s) \\
\hline$X_{0}$ & rice moisture content used in initial $\mathrm{DoE}(0.3$ d.b.) \\
\hline$X_{3}$ & $\begin{array}{l}\text { Pfost moisture content at which the logarithm dif- } \\
\text { ferentiation of water activity in temperature is }-1 \\
(0.2873 \text { d.b.) }\end{array}$ \\
\hline$X_{4}$ & $\begin{array}{l}\text { Pfost moisture content at which the logarithm dif- } \\
\text { ferentiation of water activity in temperature is } \\
-\exp (1)(0.2380 \text { d.b.) }\end{array}$ \\
\hline$Y_{\max }$ & $\begin{array}{l}\text { maximal moisture content of air of equipment } \\
\left(0.3 g_{v a} g_{d a}^{-1}\right)\end{array}$ \\
\hline$Y_{\min }$ & $\begin{array}{l}\text { moisture content of far air atmosphere } \\
\left(0.005 g_{v a} g_{d a}^{-1}\right)\end{array}$ \\
\hline$\rho_{d m}$ & $\begin{array}{l}\text { density of dry matter of rice }[10, \text { p. 305] } \\
\left(1494.2 \mathrm{~kg} / \mathrm{m}^{3}\right)\end{array}$ \\
\hline$\sigma_{y}$ & $\begin{array}{l}\text { standard deviation of measurement of moisture } \\
\text { content Section } 3.2(0.005 \text { d.b.) }\end{array}$ \\
\hline
\end{tabular}

In addition, in review [4], one can find some papers where nonLP models are integrated numerically, just like food models need to be. They all use DoEL methods, unfortunately most of them are not experimentally validated. Finally, there is few works available where models are non-LP, with numerical integration (i.e. no analytical solution available) and true experimental validation. These latter papers can be split in two categories: 
- Some papers are dealing with very specific DoEL methods like trying to design sampling locations [5] or to identify one single concentration [6] with a dedicated methodology.

- Others are based on a global optimization algorithm $[7,8]$ leading to extremely numerous simulations (hundreds of thousands [9]), hence prohibitive computations.

In our applicative example (drying), the model [10] is non-LP and does not admit any analytical solution. DoEL methods (Aoptimality, D-optimality, and E-optimality) are used. To avoid the cumbersome global optimization algorithms, the model parameters and the protocol parameters are reparameterized [11-15], allowing - in practice - the use of local optimizers leading to only thousands of simulations to reduce the computation time of the estimation. In our case, numerous various initial guesses are randomly picked and given to the deterministic optimization algorithm for more robust results.

The random excitation community knows that any varying input is quite always better for parameter estimation than constant inputs [16]. Hence, it is surprising that the usual method for drying models identification consists in carrying out many drying experiments (kinetics) each under different, but constant, drying conditions.

A short survey on standard experimental strategies found in publications in the field covered by the Drying Technologies Journal, focusing on numbers 10 and 11 of 2009 issue, shows that 16 articles contain a two-factor experimental design for 4-28 experiments. For instance [17, p. 1128] contains 12 experiments for ginger drying. None of these designs are mathematically related to a drying model, even a polynomial one. The median number of experiments is 9, in relation to the 'two-factor and three-level' experimental design.

Only one article [18, p. 1034], contains experiments with dynamic conditions, with prescribed temperature and relative humidity depending on current water content and human knowledge (published in [19, p. 163]). One cannot speak of optimal design here.

In this work, we present sequential DoEL of dynamic models [20] applied to drying; the originality of this work is the construction of a detailed methodology including reparameterization to apply DoEL to a drying dynamic model. This methodology implements, as a Matlab toolbox, the A-, D- and E-optimality on the estimation of 5 unknown (transfer related) coefficients in a compartmental model used to describe the convective drying of rice. This model, used in commercial simulators, contains strong physical phenomena couplings and non-linearities. Our method can take into account all technological constraints to calculate the best non-constant drying conditions to obtain the smallest uncertainties on estimated parameters at the lowest experimental cost. First experiment is thus optimized on the basis on an initial (plausible) guess of unknown parameters while further identified parameters are used to optimize the next experiment. In the methodology of sequential DoEL, the parameter vector $\theta^{(i)}$ is identified after each experiment $\# i$. Hence the succession of parameter vectors $\theta^{(1)}, \theta^{(2)}, \theta^{(3)}$, ... form a sequence expected to converge rapidly (usually in 2-3 Exp.) to true values with smallest possible uncertainties.

The aim of this work is to demonstrate to the process community that a single (or a few) experiments under non-constant conditions can do a better job than classical strategy based on many experiments under constant conditions to achieve low uncertainties on estimated parameters. This implies far less experimental work, on much shorter global duration (e.g. 1 day instead of 3 ).

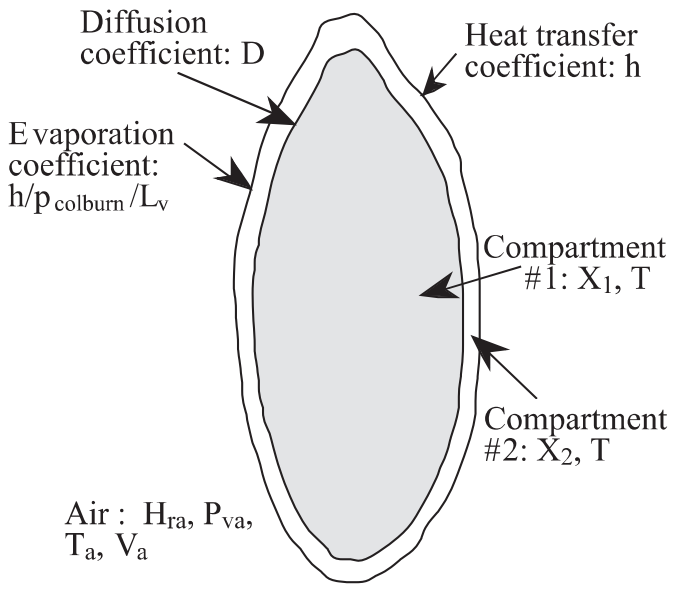

Fig. 1. Two-compartment formalism of rice.

\section{Theory}

\subsection{Model of rice drying}

In this work, the validated rice drying model from [10] is considered. This 2-compartment dynamic model is composed of $3 \mathrm{ODE}$ with strong non-linearities brought mostly by $a_{w}$ and $P_{\text {sat }}$ equations. The state vector is composed of inner and outer compartment moisture contents, and one global temperature. Moisture is evaporating from outer compartment. Only the mean $\bar{X}$ of the two compartment moisture contents is effectively measured online. All parameters are known, except four parameters parameterizing the transfer-related functions $h=h\left(T_{a}\right)$ (convective heat at the surface) and $D=D(T, \bar{X})$ (apparent water diffusion inside the kernel; it should be noted that in the original publication, it is presented in a slightly different formalism with water exchange coefficient), and a fifth parameter which is the volume fraction $\tau_{1}$ of inner compartment, see Fig. 1.

$\frac{d X_{1}}{d t}=\frac{D S_{\text {spec }}}{l_{1}}\left(X_{2}-X_{1}\right)$

$\frac{d X_{2}}{d t}=\frac{D S_{\text {spec }} \tau_{1}}{l_{1} \tau_{2}}\left(X_{1}-X_{2}\right)+\frac{h S_{\text {spec }}}{c_{\text {colburn }} L_{v} \rho_{d m} \tau_{2}}\left(P_{v a}-P_{v p}\right)$

$\frac{d T}{d t}=h S_{\text {spec }} \frac{T_{a}-T+\left(\left(P_{v a}-P_{v p}\right) / c_{\text {colburn }}\right)}{\rho_{d m}\left(c_{p d m}+\bar{X} c_{p w}\right)}$

The $P_{v p}$ function is a - strongly non-linear - function of the water activity $a_{w}$, thus of $X_{2}$ (moisture content of outer compartment) and $T$ (uniform temperature). It is given by

$P_{v p}=P_{s a t} a_{w}=10^{a_{3}+\left(a_{4}-a_{3}\right) T /\left(T+T_{3}\right)} a_{w}$

where the water activity $a_{w}$ follows the Pfost equation [21] with parameters identified by [22, p. 79] (see "Constants"):

$a_{w}=e^{-e^{\left(X_{3}-X_{2}\right) /\left(X_{3}-X_{4}\right)} /\left(T-T_{4}\right)}$

Although identified on paddy rice, it was preferred to the standard water activity because it was experimentally proven that this latter is wrong at some drying conditions used in this paper (i.e. for low temperatures $\left(25^{\circ} \mathrm{C}\right.$ ) and standard hygrometry (50\%)) [23]. From [22], it is assumed that the logarithm of moisture diffusivity $D$ is linear in $\bar{X} T$, and that the logarithm of the convective heat transfer $h$ is linear in $T_{a}$. Hence four unknown parameters are hidden in $D$ and $h$. A fifth and last unknown parameter is the 
volume fraction $\tau_{1}$ of inner compartment. ${ }^{1}$ The volume fraction of the outer compartment is $\tau_{2}=1-\tau_{1}$.

Hence, we have:
(2) The total vector region should be parallelipipedic (i.e. simple boundaries on each item) and each dimension should have a domain of variation whose order of magnitude is close to one.

$$
\begin{array}{r}
D(T, X)=10^{\wedge}\left(\frac{T_{\max } X_{0}-T X}{T_{\max } X_{0}} \log _{10}(D(T=0, X))+\frac{T X}{T_{\max } X_{0}} \log _{10}\left(D\left(T_{\max }, X_{0}\right)\right)\right)=D(T=0, X)^{\left(T_{\max } X_{0}-T X\right) / T_{\max } X_{0}} D\left(T_{\max }, X_{0}\right)^{T X / T_{\max } X_{0}} \\
h(T)=10^{\wedge}\left(\frac{T_{\max }-T}{T_{\max }-T_{\min }} \log _{10}\left(h\left(T_{\min }\right)\right)+\frac{T-T_{\min }}{T_{\max }-T_{\min }} \log _{10}\left(h\left(T_{\max }\right)\right)\right)=h\left(T_{\min }\right)^{\left(T_{\max }-T\right) /\left(T_{\max }-T_{\min }\right)} h\left(T_{\max }\right)^{\left(T-T_{\min }\right) /\left(T_{\max }-T_{\min }\right)}
\end{array}
$$

The mean moisture $\bar{X}$ is calculated as $\tau_{1} X_{1}+\tau_{2} X_{2}$. It is the only product related variable that is measured online. The variables that can be modified during the experiments are $T_{a}$ and $P_{v a}$, and they are also measured online.

\subsection{Prerequisites and general overview of DoEL methods}

The methodology of DoEL [24] has a number of prerequisites:

(a) A vector (a "protocol"), $\varphi$, formalizing [25] the degree of freedom in the experiments.

(b) A computer modelization ("model") of experiments taking the protocol $\varphi$ in account.

(c) An initial guess for each unknown parameter of this model, $\theta^{(0)}$.

(d) Information on the measurement error of $\bar{X}$ (standard deviations $\sigma_{y}$ ).

All above prerequisites are also prerequisites to generic parameter identification with gradient method. A general DoE method, given the above four elements ((a)-(d)), is the following [24]:

(i) Set $i$ to 1 .

(ii) Find the A-optimal protocol $\varphi_{i}$, by optimization (DoEL).

(iii) Make an experiment as close as possible to the calculated protocol $\varphi_{i}$. Collect real air conditions and measurement times in vector $\tilde{\varphi}_{i}$, and collect measurements $\tilde{\bar{X}}\left(\tilde{\varphi}_{i}, n_{t}\right)$ for $n_{t}=$ $1,2, \ldots, N_{\tilde{\varphi}_{i}}$.

(iv) Identify, by optimization, ith parameter vector $\theta^{(i)}$ with which the model best predicts all available measurements.

(v) Quantify the parameter confidence region and intervals around $\theta^{(i)}$. Depending on the acceptability of parameter confidence intervals, the algorithm may loop back to (ii).

A general sequential DoEL methods loops the above general DoEL method (ii) $-(v)$, once only, or a few times, as illustrated in Fig. 2.

This sequential DoEL method described in Section 2.2 and illustrated in Fig. 2 may be used on another model and on another pilot plant. Then, $\bar{X}$ should be replaced by the output of the model, $\tilde{X}$ should be replaced by an experimental measurement, and $n_{t}$ should be interpreted as an index of experimental measurement.

More details about prerequisites (a), (c) and (d) and steps (ii), (iv) and (v) are given below.

\subsection{Improved reparameterization of protocol and parameter vectors}

To allow the use of standard optimization routines, the protocol vector and the parameter vector should be reconditioned (normalization) such that:

(1) Vector elements should be chosen in order to minimize coupled effects on model predictions.

\footnotetext{
${ }^{1} \tau_{1}$ can be seen as a tuning parameter depending on the product.
}

(3) Vector should be chosen to ensure significant and smooth influence on model predictions, on the whole domain of variation.

This leads to much faster computations. This reparametrization technique is not limited to the rice drying case and can be applied to other processes; it has already been done for the parameter vector in [11, pp. 7-68, 5-13, 12].

For this application, we have chosen to model the protocol as five segments for a total of $t_{\max }=2 \mathrm{~h}$ drying. These values $(2 \mathrm{~h}$ and 5 segments) are set arbitrarily to get a compromise between performance, practicability and equipment constraints (duration of typical transitory regimes). The experimental inputs (temperature and water content of air) are parameterized (Appendix A) according to a preliminary evaluation of the real inertia of the drying pilot, with varying transition times [4, p. 4855, 26]. So, each experiment is divided into five periods. Each period has its air temperature set point $T_{a \infty i}$, relative humidity set point $H_{R a \infty i}$, and planned duration $t_{\infty i}$, with $i=1,2, \ldots, 5$, all gathered in the protocol definition $\varphi$. To allow the use of standard optimization routines, the protocol vector is reconditioned (normalization) according to rules (1)-(3) above: Hence the actual reparameterized protocol vector contains:

- The air temperature set points (divided by $T_{\max }$ for normalization) of each of the five periods.

- The set points of water addition $Y-Y_{\min }$ divided by maximum available value (saturation, or maximum capacity $Y_{\max }$ reached) for each of the five periods. These set points can be easily converted in relative humidity set points.

- The duration of each first four periods divided by remaining time of current experiment.

And the protocol writes:

$$
\begin{aligned}
\varphi= & {\left[\frac{T_{a \infty 1}}{T_{\max }}, \frac{T_{a \infty 2}}{T_{\max }}, \ldots, \frac{T_{a \infty 5}}{T_{\max }}, \ldots, \frac{Y_{1}-Y_{\min }}{\min \left(Y_{s a t}\left(T_{a \infty 1}\right), Y_{\max }\right)-Y_{\min }},\right.} \\
& \frac{Y_{5}-Y_{\min }}{\min \left(Y_{s a t}\left(T_{a \infty 5}\right), Y_{\max }\right)-Y_{\min }}, \ldots, \frac{t_{\infty 1}}{t_{\max }}, \frac{t_{\infty 2}}{t_{\max }-t_{\infty 1}}, \\
& \left.\frac{t_{\infty 4}}{t_{\max }-t_{\infty 1}-t_{\infty 2}-t_{\infty 3}}\right]
\end{aligned}
$$

We make multistage integration of the model as in [27].

\subsubsection{Definition of the parameter vector}

Using the same reasoning (see items (1)-(3) above), the five (unknown) parameters are grouped into a parameter vector as follows:

$$
\begin{aligned}
\theta= & {\left[\log _{10}(D(T=0, X)), \log _{10}\left(\frac{D\left(T_{\max }, X_{\max }\right)}{D(T=0, X)}\right),\right.} \\
& \left.\log _{10}\left(h\left(T_{\min }\right)\right), \log _{10}\left(h\left(T_{\max }\right)\right), \tau_{1}\right]
\end{aligned}
$$

The initial guess $\theta^{(0)}$ of each five parameters is collected from references shown in Table 1. 


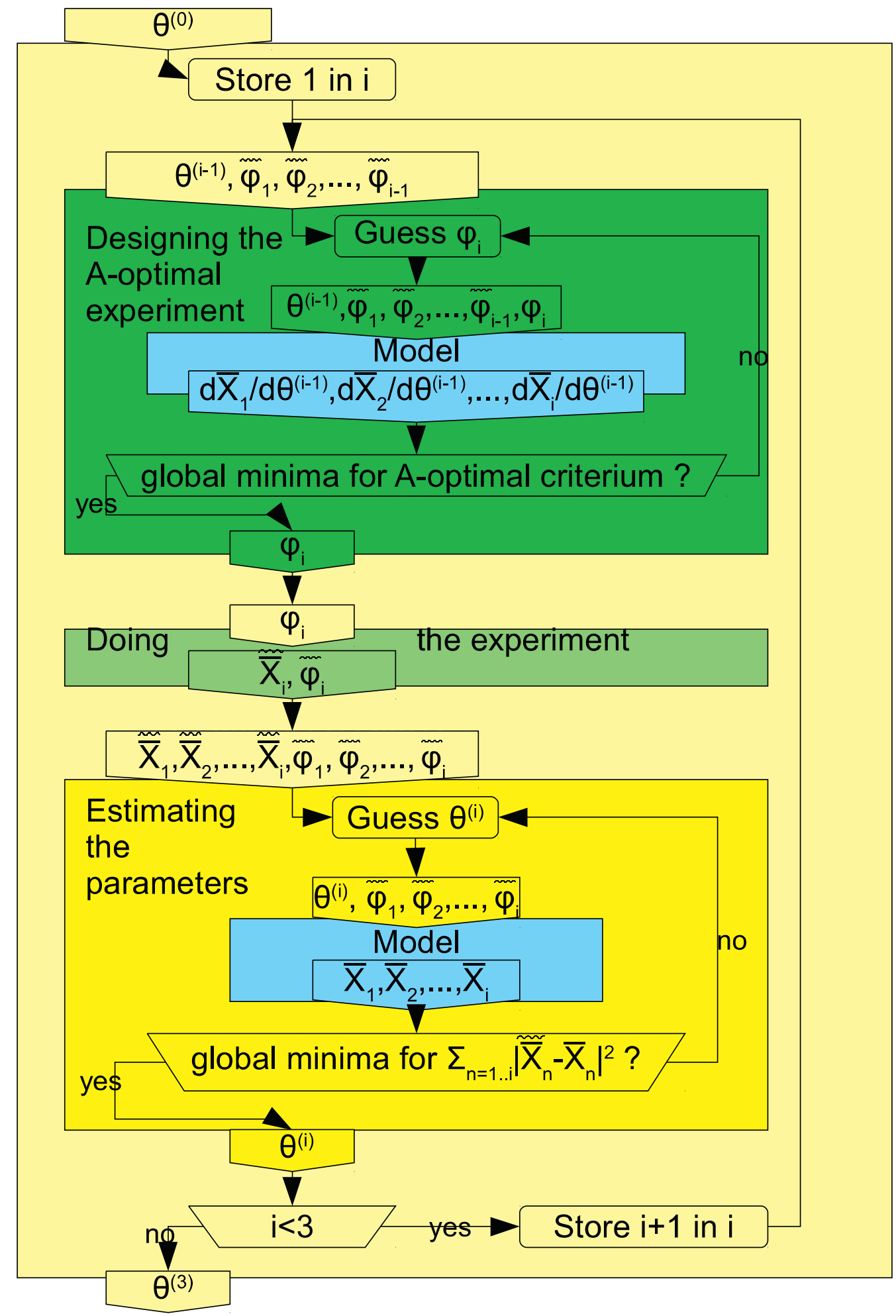

Fig. 2. Function call structure of sequential design of optimal experiment. The main loop of main function does the following: the protocol vector $\varphi_{i}$ is optimized, then the corresponding experiment is done, giving experimental values $\tilde{\bar{X}}_{i}$, then the parameter vector $\theta^{(i)}$ is estimated. $\bar{X}_{i}$ is a shorthand for the vector $\bar{X}\left(\theta, \varphi_{i}, 1\right), \bar{X}\left(\theta, \varphi_{i}, 2\right), \ldots, \bar{X}\left(\theta, \varphi_{i}, N_{\varphi_{i}}\right)$ containing the measures of mean moisture in experiment $\varphi_{i}$. The stopping criterion is selected as $i=3$, it could be replaced by something based on the acceptability of parameter confidence intervals to the operator of the experiment.

\subsection{Confidence regions and intervals}

It is important to describe how the prerequisite (d) of Section 2.2 , i.e. knowing measurement errors, is used in point $(v)$ of Section 2.2 to quantify the acceptability of parameter confidence. For that purpose, in the DoEL methods, the sensitivity (or Jacobian) matrix, $M_{\text {Jacobian, }}$ and the Fisher information matrix, $M_{\text {Fisher }}$ must be defined.

The sensitivity matrix, $M_{\text {Jacobian }}$ gives the sensitivities of the model prediction in the parameter vector around a value of this 
Table 1

Ranges and initial guesses for parameters, collected from publications

\begin{tabular}{llllll}
\hline Parameter & Initial & Minimal & Maximal & Unit & Reference for initial guess \\
\hline$D(T=0, X)$ & 4.8771 & $10^{-3}$ & $10^{6}$ & $\mu \mathrm{m}^{2} / \mathrm{s}$ & [28, p. 330, Table 2, $\tau=0]$ at 24\% d.b. \\
$D\left(T_{\max }, X_{0}\right)$ & 79.2984 & 10 & $10^{6}$ & $\mu \mathrm{m}^{2} / \mathrm{s}$ & [29, p. 144, Eq. (2), p. 145, Eq. (6), p. 151] at 40 ${ }^{\circ} \mathrm{C}$ and 40\% d.b. \\
$h\left(T_{\min }\right)$ & 11.336 & 1 & 200 & $\mathrm{~W} / \mathrm{Km}^{2}$ & {$[30,31, \mathrm{p} .521]$ at 0 ${ }^{\circ} \mathrm{C}$,} \\
$h\left(T_{\max }\right)$ & 10.0551 & 1 & 200 & $\mathrm{~W} / \mathrm{Km}^{2}$ & [30, 31, p. 521] at 100 ${ }^{\circ} \mathrm{C}$, \\
$\tau_{1}$ & 0.8225 & 0 & 1 & $\mathrm{~m}^{3} / \mathrm{m}^{3}$ & [32, p. OP173]. \\
\hline
\end{tabular}

parameter $\theta$. It is a rectangular matrix, whose coefficient line $i$ col$\mathrm{umn} j$ is the differentiation by $j$ th parameter of the model prediction of the $i$ th available and ongoing design experimental data. In the design of the third experiment, this matrix $M_{\text {Jacobian }}$ is:

$$
M_{\text {Jacobian }}=\left(\begin{array}{cccc}
\frac{d \bar{X}}{d \theta_{1}}\left(\theta^{(2)}, \tilde{\varphi}_{1}, 1\right) & \frac{d \bar{X}}{d \theta_{2}}\left(\theta^{(2)}, \tilde{\varphi}_{1}, 1\right) & \cdots & \frac{d \bar{X}}{d \theta_{5}}\left(\theta^{(2)}, \tilde{\varphi}_{1}, 1\right) \\
\frac{d \bar{X}}{d \theta_{1}}\left(\theta^{(2)}, \tilde{\varphi}_{1}, 2\right) & \frac{d \bar{X}}{d \theta_{2}}\left(\theta^{(2)}, \tilde{\varphi}_{1}, 2\right) & & \frac{d \bar{X}}{d \theta_{5}}\left(\theta^{(2)}, \tilde{\varphi}_{1}, 2\right) \\
\vdots & \vdots & & \vdots \\
\frac{d \bar{X}}{d \theta_{1}}\left(\theta^{(2)}, \tilde{\varphi}_{1}, N_{\tilde{\varphi}_{1}}\right) & \frac{d \bar{X}}{d \theta_{2}}\left(\theta^{(2)}, \tilde{\varphi}_{1}, N_{\tilde{\varphi}_{1}}\right) & \cdots & \frac{d \bar{X}}{d \theta_{5}}\left(\theta^{(2)}, \tilde{\varphi}_{1}, N_{\tilde{\varphi}_{1}}\right) \\
\frac{d \bar{X}}{d \theta_{1}}\left(\theta^{(2)}, \tilde{\varphi}_{2}, 1\right) & \frac{d \bar{X}}{d \theta_{2}}\left(\theta^{(2)}, \tilde{\varphi}_{2}, 1\right) & \cdots & \frac{d \bar{X}}{d \theta_{5}}\left(\theta^{(2)}, \tilde{\varphi}_{2}, 1\right) \\
\frac{d \bar{X}}{d \theta_{1}}\left(\theta^{(2)}, \tilde{\varphi}_{2}, 2\right) & \frac{d \bar{X}}{d \theta_{2}}\left(\theta^{(2)}, \tilde{\varphi}_{2}, 2\right) & & \frac{d \bar{X}}{d \theta_{5}}\left(\theta^{(2)}, \tilde{\varphi}_{2}, 2\right) \\
\vdots & \vdots & & \vdots \\
\frac{d \bar{X}}{d \theta_{1}}\left(\theta^{(2)}, \tilde{\varphi}_{2}, N_{\tilde{\varphi}_{2}}\right) & \frac{d \bar{X}}{d \theta_{2}}\left(\theta^{(2)}, \tilde{\varphi}_{2}, N_{\tilde{\varphi}_{2}}\right) & \cdots & \frac{d \bar{X}}{d \theta_{5}}\left(\theta^{(2)}, \tilde{\varphi}_{2}, N_{\tilde{\varphi}_{2}}\right) \\
\frac{d \bar{X}}{d \theta_{1}}\left(\theta^{(2)}, \varphi_{3}, 1\right) & \frac{d \bar{X}}{d \theta_{2}}\left(\theta^{(2)}, \varphi_{3}, 1\right) & \cdots & \frac{d \bar{X}}{d \theta_{5}}\left(\theta^{(2)}, \varphi_{3}, 1\right) \\
\vdots & \vdots & & \vdots \\
\frac{d \bar{X}}{d \theta_{1}}\left(\theta^{(2)}, \varphi_{3}, N_{\varphi_{3}}\right) & \frac{d \bar{X}}{d \theta_{2}}\left(\theta^{(2)}, \varphi_{3}, N_{\varphi_{3}}\right) & \cdots & \frac{d \bar{X}}{d \theta_{5}}\left(\theta^{(2)}, \varphi_{3}, N_{\varphi_{3}}\right)
\end{array}\right)
$$

The model Eqs. (1)-(3) is defined by a set of 3 ODEs. Using the directdifferentiation method $[33,34,5$, p. 29] the sensitivity matrix can be computed by numerical integration of a set of 18 ODEs: the original 3 ODEs Eqs. (1)-(3) and their differentiation in the 5 parameters. For example, the fourth equation is the differentiation of Eq. (1) with respect to $\theta_{1}$, namely:

$\frac{d\left(d X_{1} / d \theta_{1}\right)}{d t}=\frac{\left(\partial D / \partial \theta_{1}\right) S_{\text {spec }}}{l_{1}}\left(X_{2}-X_{1}\right)+\frac{D S_{\text {spec }}}{l_{1}}\left(\frac{d X_{2}}{d \theta_{1}}-\frac{d X_{1}}{d \theta_{1}}\right)$

The Fisher information matrix, $M_{\text {Fisher }}$, is a matrix, which can be computed by

$M_{\text {Fisher }}=M_{\text {Jacobian }}^{T} \times \Sigma^{-1} \times M_{\text {Jacobian }}$

where $\Sigma$ is the variance/covariance matrix, here a diagonal matrix (because we assume that the noise of measures are independent), whose coefficient line $i$ is the variance of the $i$ th available measures (actually recorded in past experiments, or currently planned), which is prerequisite (d) (see Section 2.2). Confidence intervals and confidence regions can be computed using this Fisher information matrix $M_{\text {Fisher }}$.

The confidence region of the parameter is an ellipsoid, centered on the parameter vector, whose axis are the eigenvectors of the Fisher information matrix, and whose axis lengths are proportional to the square-root of the inverse of the respective eigenvalues [35]. As we have five parameters in this article, the proportion coefficient is 6.654 since it is twice the square root of $\chi^{2}(5)$ value at 95\%(cumulated normal distribution with probability $95 \%$ and 5 degrees of freedom [36, Section 10.2, p. 144]).

The specific confidence interval length of each estimated parameter is proportional to the square root of inverse of the diagonal coefficients of the Fisher information matrix [24]. This proportion coefficient is 3.92 since it is twice the square root of the product of the $\chi^{2}(1)$ value at $95 \%$.

The introduction stated that DoENL methods give more precise result but for bigger computation times than DoEL methods. Hence we used DoEL methods, and the confidence regions and confidence intervals are only valid asymptotically (when the time interval between two weighting $t_{6}$ is supposed very small) due to the nonlinearity of the model. Hence sometimes the confidence intervals do not contain the final value.

\subsection{Optimization}

\subsubsection{Step (ii): optimizing the experimental protocol $\varphi$}

The A-, D- and E-optimal criteria are, respectively, the trace, the determinant, and the spectral radius [24] of the matrix inverse of the Fisher information matrix. In the last two of them, the matrix inversion may be bypassed, when its computation time overhead on simulation model is not negligible. For most ODE problems, this overhead is way negligible. A protocol is A-, D- and E-optimal when the respective criterion is minimal. To overcome possible over-influence of some matrix coefficient and ensure that a nearly singular information matrix does not give undue advantage to a protocol vector, the inversion is re-balanced by adding $\pm 3 \times 10^{-13}$ or $3 \times 10^{-11} \%$ to its matrix coefficient. This coefficient is selected according to the fact it maximizes the A-, D- or E-optimal criterion of DoEL. The optimal protocol $\varphi$ is computed using a non-linear optimization routine according to the selected criterion.

For example, during the real protocol design, before experiment number 3 , the protocol $\varphi_{3}$ was A-optimally designed by minimizing the trace of the re-balanced inverse of the Fisher information matrix, which is a $5 \times 5$ matrix whose element line $n_{l}$ and column $n_{c}$ is

$$
\begin{aligned}
& \frac{1}{\sigma_{y}^{2}} \sum_{n_{e}=1}^{2} \sum_{n_{t}=1}^{N_{\tilde{\varphi}_{n}}} \frac{\partial \bar{X}\left(\theta^{(2)}, \tilde{\varphi}_{n_{e}}, n_{t}\right)}{\partial \theta_{n_{c}}} \times \frac{\partial \bar{X}\left(\theta^{(2)}, \tilde{\varphi}_{n_{e}}, n_{t}\right)}{\partial \theta_{n_{l}}} \\
& \quad+\sum_{n_{t}=1}^{1+t_{\max } / t_{6}} \frac{1}{\sigma_{y}^{2}} \frac{\partial \bar{X}\left(\theta^{(2)}, \varphi_{3}, t_{6} n_{t}-t_{6}\right)}{\partial \theta_{n_{c}}} \times \frac{\partial \bar{X}\left(\theta^{(2)}, \varphi_{3}, t_{6} n_{t}-t_{6}\right)}{\partial \theta_{n_{l}}}
\end{aligned}
$$

\subsubsection{Step (iv): identifying model parameters $\theta$}

A parameter $\theta$ is considered to be identified when it is found to be the only one minimizing the criterion of identification. This criterion is the least square criterion $\left(M_{r} \times \Sigma^{-1} \times M_{r}^{T}\right)^{1 / 2}$, where $M_{r}$ is a line vector whose $j$ th coefficient contains the difference between $j$ th available experimental measure, and its model prediction. The square of criterion to identifying $\theta^{(3)}$ is

$$
\frac{1}{\sigma_{y}^{2}} \sum_{n_{e}=1}^{3} \sum_{n_{t}=1}^{N_{\tilde{\varphi}_{n_{e}}}} \bar{X}\left(\theta^{(3)}, \tilde{\varphi}_{n_{e}}, n_{t}\right)-\left.\tilde{\bar{X}}\left(\theta^{(3)}, \tilde{\varphi}_{n_{e}}, n_{t}\right)\right|^{2}
$$

The initial guess $\theta^{(0)}$ shown in Table 1 is retained as an initial guess for the first estimation only. The experimentally measured temperature $T_{a}$ and water content of air (expressed as a (partial) pressure $P_{v a}$ of vapour in air) are interpolated (by Matlab's interp1) and replaced equations Eqs. (A.1) and (A.2) of Appendix 
A. The estimated parameter vector $\theta^{(i-1)}$ is retained as an initial guess for next DoE and estimation.

\section{Materials and methods}

\subsection{Rice samples}

Rewetting of rice was done with adding all required water at once to the rice in a big sealed container, followed immediately (and also one day after) by the slow rotation of the container for $1 \mathrm{~h}$. The rice container is stored in a cooled place, except for the last hour before experimentation where it is stored at $20^{\circ} \mathrm{C}$. Just before experimentation, the water content of a rewetted rice sample is measured [37, p. 73]; the subsequent DoEs take this measure in account. This measure is not available for initial planification, which used instead $X_{0}=30 \%$ d.b. as the best guess.

\subsection{Experimental dryer}

An experimental dryer is installed at the INRA's associated laboratory at AgroParisTech (Fig. 3). The inlet air temperature, relative humidity and velocity are controlled by three independent PID controllers, under the supervision of the master PC. Velocity is fixed to $1 \mathrm{~m} / \mathrm{s}$. Product (rice here) is weighted every minute (depending on the computer settings) by diverting the air flow with the air switch and releasing the pneumatic jacks supporting the basket over the balance. The current mean moisture content can be computed from this weight; it is the only product related variable that is measured online.

Besides its numerous advantages, this type of dryer presents some drawbacks for this study:

- Due to its strong thermal inertia, one cannot expect sharp changes in air temperature (e.g. Table "Constants" defines in $t_{3}$ the lag between set point change and actual change).

- Due to the presence of moving parts, it is impossible to guaranty good thermal insulation everywhere, leading to water leakage wherever humid air may saturate in contact of ambient air.

- Due to steam injection prior to final temperature adjustment, $100 \%$ of air relative humidity is unreachable.

$\sigma_{y}$ is retained for the experimental measurement error, corresponding to a load of $100 \mathrm{~g}$ of dry matter of rice for each experiment, and a standard deviation error weighting of $0.5 \mathrm{~g}$ on measurements estimated from the actual performance of the weighting device.

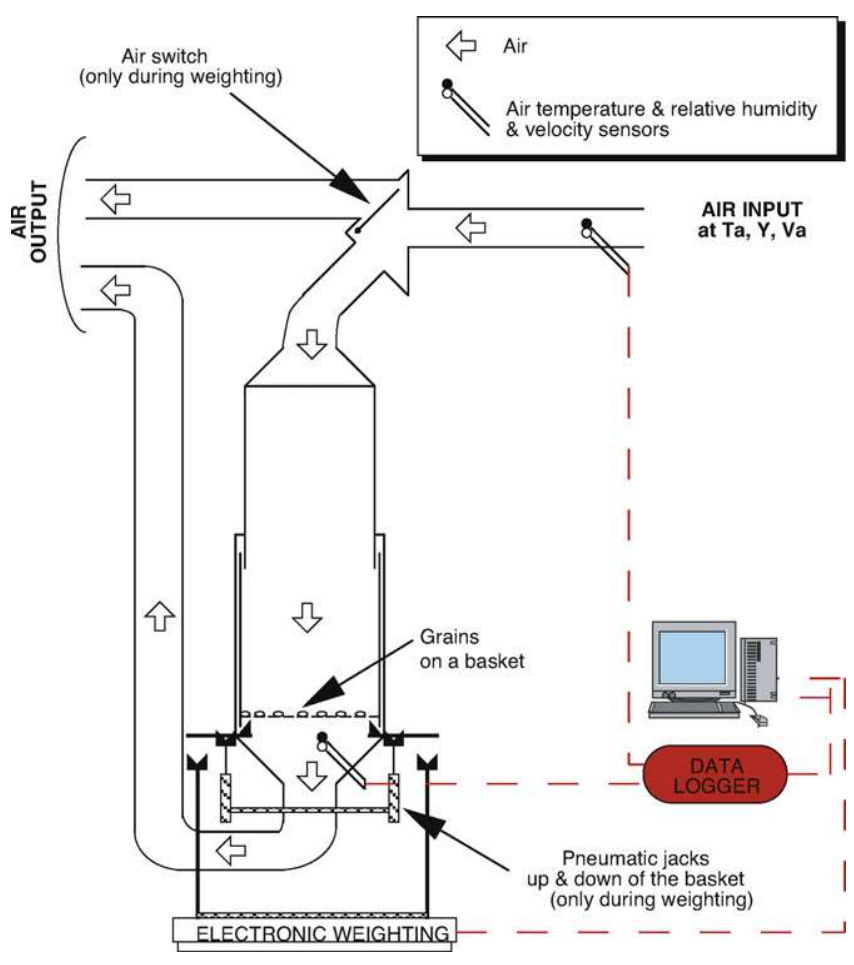

Fig. 3. Experimental dryer scheme INRA-AgroParisTech in Massy, France.

\subsection{Technological constraints on the experimental device.}

The equipment constraints on the coefficients of the protocol vector Eq. (6) are the following: Minimal value is $T_{\min } / T_{\max }$ for first 5 protocol coefficients, and 0 for last 9 protocol coefficients. Maximal value for all protocol coefficients is 1 .

The control of air drying conditions is done by PID control set points, according to the vector protocol. Hence, it is necessary to inject, in the model, the pilot inertias in response of set point changes to mimic correctly in the simulation what will happen in real.

The inertias of the air temperature and relative humidity are evaluated with $15 \mathrm{~h}$ of experiments in dynamic conditions, see Fig. 4 and discussion in Appendix A. This evaluation gives a model for inertia, detailed in Appendix A. It should be noted that this is just an empirical approximation of pilot dynamics but it is assumed to be sufficient for the extrapolation of calculated protocol to actual
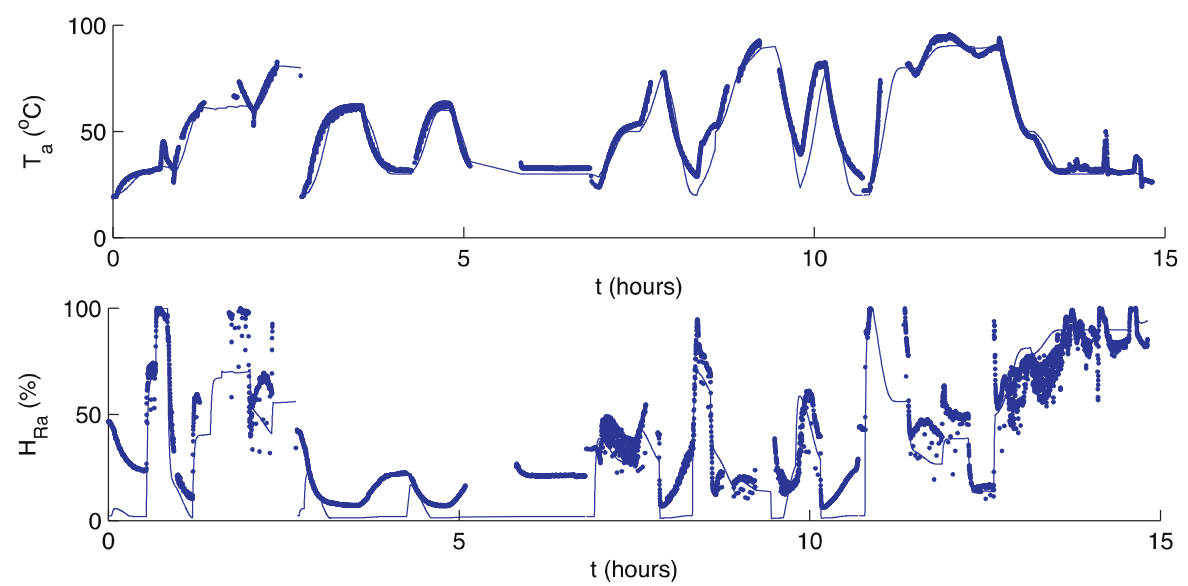

Fig. 4. Comparison between measured (.) and predicted (-) (from set points values by model in Appendix A) inertias of air relative humidity (above) and temperature (below) of variable drying conditions. 
Table 2

Convergence of DoE with simulated experiments. (a) A-optimal, (b) D-optimal and (c) E-optimal criterion is used; true parameters used in simulated experiments are displayed on (d) (these simulated experiments are placeholders for step (iv) of Section 2.2). Last column gives $\sigma_{f} / \sigma_{y}$ which is the mean lack of fit $\sigma_{f}$, divided by the mean experimental error $\sigma_{y}$. When a confidence interval covers several orders of magnitude without containing 0 , only its bounds are shown, in the form [x,y]

\begin{tabular}{|c|c|c|c|c|c|c|}
\hline Number of experiments done & $D(T=0, X)\left(\mu \mathrm{m}^{2} / \mathrm{s}\right)$ & $D\left(T_{\max }, X_{0}\right)\left(\mu \mathrm{m}^{2} / \mathrm{s}\right)$ & $h\left(T_{\min }\right)\left(\mathrm{W} / \mathrm{Km}^{2}\right)$ & $h\left(T_{\max }\right)\left(\mathrm{W} / \mathrm{Km}^{2}\right)$ & $\tau_{1}\left(\mathrm{~m}^{3} / \mathrm{m}^{3}\right)$ & $\sigma_{f} / \sigma_{y}$ \\
\hline \multicolumn{7}{|c|}{ (a) A-optimal design, simulation run } \\
\hline 1 & $39 \pm 25$ & $468 \pm 10^{9}$ & $1.05 \pm 400$ & {$[1,12,000]$} & $0.63 \pm 0.16$ & 1.0592 \\
\hline 2 & $31 \pm 3$ & {$[25,9500]$} & $11.9 \pm 1.7$ & $96.2 \pm 2.1$ & $0.61 \pm 0.025$ & 1.0873 \\
\hline 3 & $31 \pm 2$ & {$[85,2500]$} & $11.6 \pm 1.1$ & $110.456 \pm 1.8$ & $0.618 \pm 0.017$ & 1.02 \\
\hline \multicolumn{7}{|c|}{ (b) D-optimal design, simulation run } \\
\hline 1 & $10 \pm \infty$ & {$\left[10,10^{6}\right]$} & {$[1,200]$} & {$[1,200]$} & $0.63 \pm 0.2$ & 1.0272 \\
\hline 2 & $33 \pm 4$ & {$[10,26,500]$} & $10.9 \pm 0.8$ & $91 \pm 1.1$ & $0.60 \pm 0.02$ & 1.07 \\
\hline 3 & $34 \pm 2.6$ & {$[20,8200]$} & $10.9 \pm 0.6$ & $85 \pm 1$ & $0.591 \pm .013$ & 1.02 \\
\hline 4 & $31.6 \pm 2$ & {$[60,3500]$} & $11 \pm 0.4$ & $88.1 \pm 0.7$ & $0.60 \pm 0.01$ & 1.02 \\
\hline \multicolumn{7}{|c|}{ (c) E-optimal design, simulation run } \\
\hline 1 & $36 \pm 11$ & $405 \pm 10^{6}$ & $10.6 \pm 0.8$ & $91 \pm 1$ & $0.56 \pm 0.06$ & 1.0402 \\
\hline 2 & $32 \pm 3$ & {$[26,6000]$} & $10.6 \pm 0.5$ & $105 \pm 1$ & $0.57 \pm 0.03$ & 0.98 \\
\hline 3 & $32 \pm 2$ & {$[70,2550]$} & $10.8 \pm 0.5$ & $99.1 \pm 0.8$ & $0.59 \pm 0.02$ & 1.03 \\
\hline \multicolumn{7}{|c|}{ (d) Parameter expected for simulation runs } \\
\hline$\infty$ & 31.4361 & 445.2138 & 11.0314 & 100.433 & 0.6 & 1 \\
\hline
\end{tabular}

experiment. This assumption will be proved to be correct in Section 4.2 .

In addition, the experiments in which the minimal difference between rice temperature and dew point temperature is less than $10^{\circ} \mathrm{C}$ are forbidden, to ensure that unexpected condensation on an exposed part of the weighting system would not disturb experiment and increase this weighting error.

\subsection{Optimization setups}

\subsubsection{For estimation of parameter $\theta$ (as in step (iv) Section 2.2)}

The estimated parameters are found by Nelder-Mead optimization [38]. They are further enhanced with Levenberg-Marquardt optimization [39], with a maximal number of iterations set to 4000 . To avoid local minima, numerous (10) various initial guesses for parameter vector are randomly picked, leading to lengthy computation times.

\subsubsection{For design of protocol $\varphi$ (as in step (ii) Section 2.2)}

Due to the intrinsic 'discrete' nature of the $\bar{X}$ measurement (assessed every minute), the criterion for the optimization algorithm is clearly non-smooth and easily brings local minima. Some of these local minima correspond to weight measurements occurring seconds after a set point change.

The experiments in which the difference between rice temperature and dew point temperature is less than $10^{\circ} \mathrm{C}$ are forbidden according to Section 3.3. To enforce this constraint, the design criterion must be modified, such that, the sooner this rule is not satisfied, the bigger the criterion is.

So, before the real DoEL, a fake DoEL was made, with 21 randomly chosen initial protocol guesses. This fake DoEL assumed that:

(f1) The weighing aborted its one-minute-sleep at each set points change, to ensure that no weighing occurred during the $60 \mathrm{~s}$ after each set points change.

(f2) A penalty was added to ensure that, the sooner the difference between rice temperature and dew point temperature is less that $10^{\circ} \mathrm{C}$, the greater the design criterion is.

The penalty is useful to let the optimizing routine find experiments in which this constraint is satisfied.

The 21 results of these fake DoEs were then used as initial guesses for the real protocol design.

For example, during the fake protocol design, summarizing elements explained in Section 3.4, before experiment number $i=1 \ldots 3$, the protocol $\varphi_{i}$ was A-optimally designed by minimizing the sum of the penalty and of the trace of the re-balanced inverse of the $5 \times 5$ matrix whose element line $n_{l}$ and column $n_{c}$ is

$$
\begin{aligned}
& \frac{1}{\sigma_{y}^{2}} \sum_{n_{e}=1}^{2} \sum_{n_{t}=1}^{N_{\tilde{\varphi}_{n}}} \frac{\partial \bar{X}\left(\theta^{(2)}, \tilde{\varphi}_{n_{e}}, n_{t}\right)}{\partial \theta_{n_{c}}} \times \frac{\partial \bar{X}\left(\theta^{(2)}, \tilde{\varphi}_{n_{e}}, n_{t}\right)}{\partial \theta_{n_{l}}} \\
& \quad+\sum_{n_{s}=1}^{5} \sum_{n_{t}=1}^{1+t_{\infty n_{s} / t_{6}}} \frac{1}{\sigma_{y}^{2}} \min \left(1, \frac{t_{\infty n_{s}}}{t_{6}}-n_{t}+1\right) \frac{\partial \bar{X}\left(\theta^{(2)}, \varphi_{3}, t_{6} n_{t}-t_{6}+\sum_{n_{r}=1}^{n_{s}} t_{\infty n_{r}}\right)}{\partial \theta_{n_{c}}} \\
& \times \frac{\partial \bar{X}\left(\theta^{(2)}, \varphi_{3}, t_{6} n_{t}-t_{6}+\sum_{n_{r}=1}^{n_{s}} t_{\infty n_{r}}\right)}{\partial \theta_{n_{l}}}
\end{aligned}
$$

The design loop (ii)-(v) of Section 2.2 was stopped after three iterations in this paper due to practical constraints. The Nelder-Mead algorithms were configured with a maximal number of iteration set to 2000. Both Nelder-Mead and Levenberg-Marquardt algorithms were configured to stop when objective and protocol/parameter changes are less than the machine precision.

\section{Results}

The disadvantage of experiments with non constant conditions compared to experiments under constant conditions is on the way higher computation time required: $3 \mathrm{~h}$ on a cluster of 12 processors (about a day on one processor) to run on average 290,000 times the drying model. The estimation only ran on average $3000 \times i$ times the drying model, where $i$ is the number of realized experiment.

\subsection{Pure simulation run of DoEL}

The first objective here is to choose between A-, D- and E-optimality, and to check the feasibility (mostly as a quick error-checking procedure) at no experimental cost. The experimental data are replaced here by simulations with random added noise (using standard deviation $\sigma_{y}$ ) on measures predicted with another set of parameters shown in Table 2(d), named true parameters. These true parameters are the expected results of the DoE algorithm in this simulated environment.

In these favorable conditions, as shown in Table 2(a), the A-optimal algorithm converges after only 3 - simulated - experiments while D-optimality performs significantly worse in terms of quantity of bad confidence intervals than A-optimality and E-optimality.

On the basis of this pure simulation run, A-optimality is preferred to E-optimality because A-optimal criterion is smoother than the E-optimal criterion [5, p. 293]. It is not a generic conclusion, and this conclusion is also debatable because we used DoEL methods instead of DoENL methods.

\subsection{DoE confronted to experiment}

The same methodology of sequential design was then applied in a real experimental setup.

Step \#1. After the simulation validation, the A-optimal protocol used in first line of Table 2(a) is actually used on the experimental pilot, leading to data and to a new set of estimated parameters (shown in first line of Table 3(a)). 
Table 3

Experiment design convergence. Test with experiments done on drying pilot plant. (a) A-optimal design in three iterative steps and (b) standard two-factor three-level design. Last column gives $\sigma_{f} / \sigma_{y}$ which is the lack of fit $\sigma_{f}$, divided by the mean experimental error $\sigma_{y}$. When a confidence interval covers several orders of magnitude without containing 0 , only its bounds are shown, in the form $[x, y]$

\begin{tabular}{|c|c|c|c|c|c|c|}
\hline \# Exp. done & $D(T=0, X)\left(\mu \mathrm{m}^{2} / \mathrm{s}\right)$ & $D\left(T_{\max }, X_{0}\right)\left(\mu \mathrm{m}^{2} / \mathrm{s}\right)$ & $h\left(T_{\min }\right)\left(\mathrm{W} / \mathrm{Km}^{2}\right)$ & $h\left(T_{\max }\right)\left(\mathrm{W} / \mathrm{Km}^{2}\right)$ & $\tau_{1}\left(\mathrm{~m}^{3} / \mathrm{m}^{3}\right)$ & $\sigma_{f} / \sigma_{y}$ \\
\hline \multicolumn{7}{|c|}{ (a) A-optimal design, experimental run } \\
\hline 1 & $20 \pm 5$ & $100 \pm \infty$ & {$[3,20]$} & {$[6,23]$} & $.55 \pm 0.02$ & 1.09 \\
\hline 2 & $7.5 \pm 0.9$ & {$[135,5500]$} & {$[3,200]$} & {$[1,200]$} & $0.68 \pm 0.03$ & 1.01 \\
\hline 3 & $5.9 \pm 0.9$ & {$[200,9500]$} & $13.7 \pm 0.9$ & $11.1 \pm 0.8$ & $0.56 \pm 0.01$ & 1.34 \\
\hline \multicolumn{7}{|c|}{ (b) two-factor three-level design, experimental run } \\
\hline 9 & $6.9 \pm 0.5$ & {$[180,3200]$} & $6.5 \pm 0.5$ & $13.0 \pm 0.5$ & $0.69 \pm 0.01$ & 1.74 \\
\hline
\end{tabular}

Step \#2. Then, a second A-optimal protocol of $2 \mathrm{~h}$ is designed, taking in account these parameters, and then applied in the drying lab, leading to more data and to another set of estimated parameters (see second line of Table 3(a)).

Step \#3. Then, a third A-optimal protocol of $2 \mathrm{~h}$ is designed and then applied in the drying lab, leading to more data and to the final parameter set with acceptable confidence intervals (see last line of Table 3(a)).

The discrepancies shown in Fig. 5 between designed and actual protocols can be explained by the difficulty to integrate technological constraints in the optimization procedure. Indeed, there are strong couplings between the increase of the RH by steam addition and temperature control (steam is heating air very fast), and the dynamics of their inertia are, overall, predicted by their model shown in Appendix A.

It is observed, in general, that the computed A-optimal protocol tends to maximize the amplitude of the variations of air drying conditions. The drier the product gets, the longer the segment tends to be to compensate for smaller mass variations. The experimentally obtained drying conditions share the same characteristics, despite the above discrepancies: hence, even if they are not exactly equal to optimal designs, they nonetheless give a similar amount of information on average, according to the A-optimal criterion. This validates the model of inertia (see Appendix A), which is precise enough for DoEL methods. In addition, it is observed that first experiment reaches directly the right parameter neighborhood in terms of order of magnitude.

Fig. 6(a)-(c) displays both the simulated and the actual mean moisture content, for the three successive experiments. Fig. 6(a) clearly shows that, after $40 \mathrm{~min}$ of drying, there is a fast and temporary unmatched increase of water content, which may be due to condensation (at the same time, the moisture content of air is close to saturation in Fig. 5). Despite this, the lack of fit $\left(\sigma_{v} / \sigma_{y}\right)$ is only 1.09 times bigger (see Table 4) than the measurement error, which is good. Fig. 6(b) Exp. \#1 shows again the data of first dynamic experiment, but fitted together with the data of second dynamic experiment. The influence of unmatched increase of water on the prediction seems to decrease, which is positive. Fig. 6(b) Exp. \#2 shows the data of second dynamic experiment. We can see that the experimental data of first experiment is not better fitted as in Fig. 6(a), but the predicted moisture content of outer compartment is different, reflecting that more information was gained from these two experiments. As can be observed in Fig. 6(c), the agreement is quite good after only three short drying experiments. Observed error is less than 1.4 times the measurement error on average (see Table 4), and uncertainties on identified parameters are low (see Table 3(a)).

\subsection{Validation: comparison to classical DoEs.}

\subsubsection{Step \#4: validation of prediction}

To validate the results shown in Table 3(a), the model estimated at step\#3 is used to predict the data obtained by a two-factor three-level grid of nine experiments, combining three temperature levels of $50^{\circ} \mathrm{C}, 70^{\circ} \mathrm{C}$ and $90^{\circ} \mathrm{C}$, and three levels of relative humidity $0 \%, 20 \%$ and $40 \%$.

Fig. 6(d) shows the comparison between prediction by parameter identified in step \#3 and these nine experiments. In this comparison, the mean error is 4.33 times the measurement error. Our laboratory notebook shows that a mismanipulation occurred during experiments \#6 and \#8 (leading to lower air velocities), which is the likely reason for this error. However, the value 4.33 is to be compared to 1.73 which is the minimum value it could have had.

\subsubsection{Step \#5: independent estimation}

The parameters were also estimated from the nine experiments \#4-12 in constant conditions, excluding the three dynamic conditions. The resulting predicted simulations are shown in Fig. 7(a), and respective predicted values are in Table 3(b). The observed mean error is 1.84 times the measurement error, which is fairly acceptable. The comparison of results of the two independently identified parameter sets is possible from last two lines of Table 3. Their disagreement is due to the experimental problems as stated above.

\subsubsection{Step \#6: validation of experiments with static conditions} on variable conditions

Parameters from step \#5 were used to predict the result of dynamic experiments, see Fig. 7(b). There is a slight global overestimation of the prediction of dynamics for similar reasons as stated previously.

The observed mean error of prediction is 3.25 times the measurement error according to Table 4. The cross-validation comparison between this validation and the validation of A-optimal designed experiment (see step \#4) is hence only marginally in favor of 9 experiments on static conditions, it has about $33 \%$ smaller validation errors. Thanks to the reparameterization of the parameter and protocol vectors and to the use of numerous various initial guesses in minimization algorithms, initial value of the protocol $\theta^{(0)}$ and the initial assumption of parameter $\phi_{i}$ were found to have no significant influence on the final results.

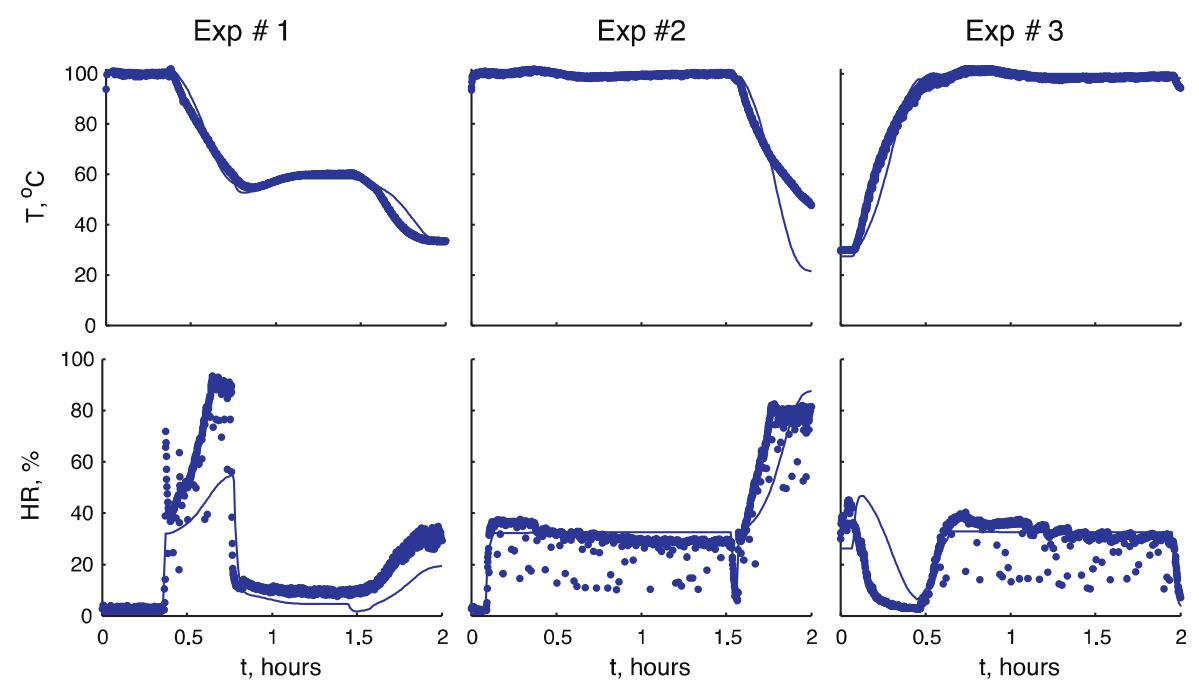

Fig. 5. Comparison between planned (-) and measured (.) air relative humidity and temperature of variable drying conditions of the three designed dynamic experiment. 

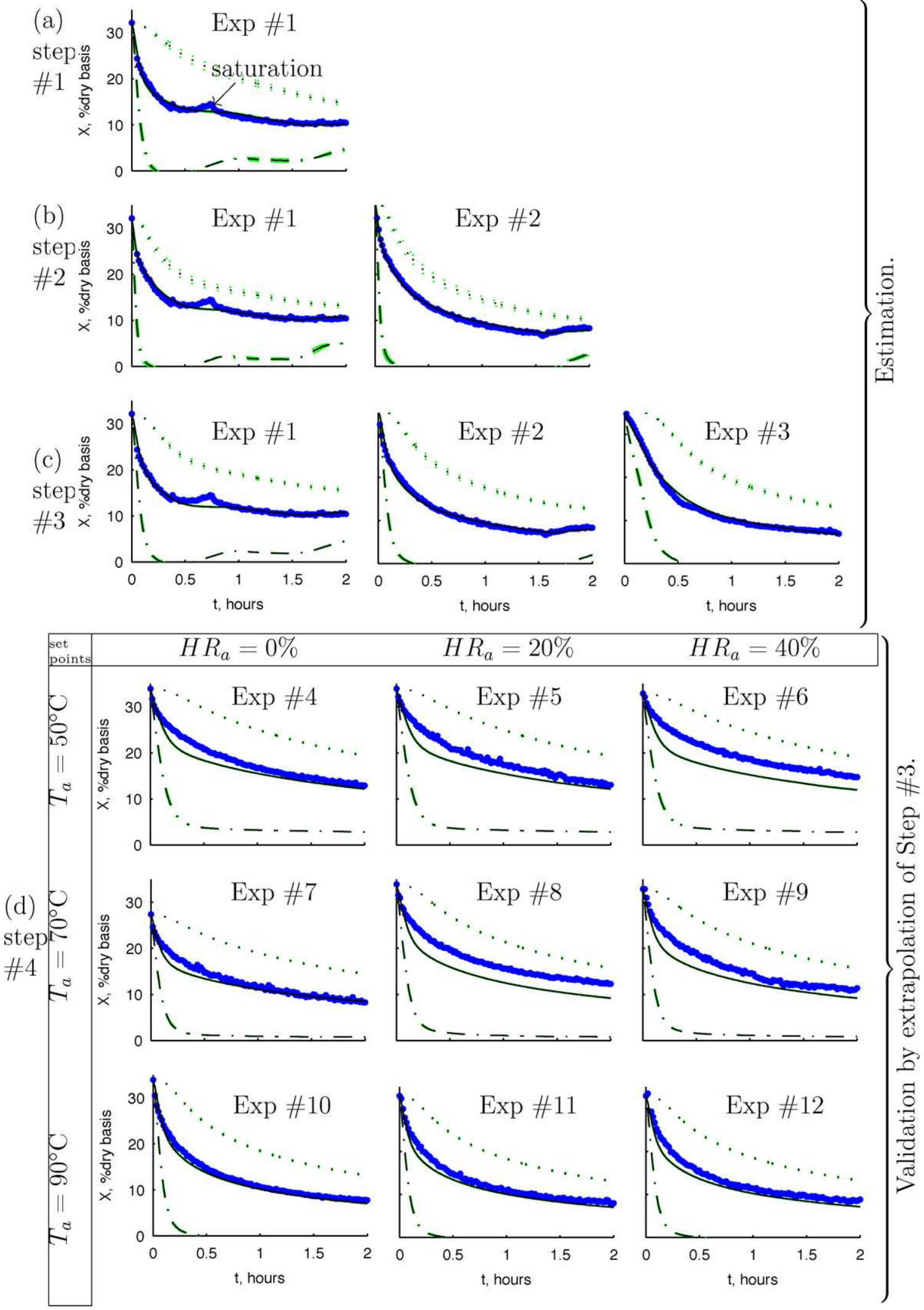

Fig. 6. Three steps of designed drying experiment, and their validation step. Mean moisture content predicted by model for inner compartment (..), outer compartment (-.); mean moisture content recorded (. in dark blue) and predicted by model (- in black). Confidence bands of predictions are in light green. Simulation at step \#1 (resp. \#2 and \#3) is based on parameters computed from only data recorded from experiment data available at these steps. Simulation at step \#4 is based from parameter computed at step \#3, hence from experiments \#1, \#2 and \#3. (For interpretation of the references to color in this figure legend, the reader is referred to the web version of this article.) 
Table 4

Experimental validation of A-optimal DoEL method: for each line of Table 3, this table details the experiments used to identify the parameter set in first four columns, and, in the same line, the experiments used to validate this same parameter set, in last four columns.

\begin{tabular}{|c|c|c|c|c|c|c|c|}
\hline \multicolumn{4}{|c|}{ Experiments used to identify the parameter } & \multicolumn{4}{|c|}{ Experiments predicted to validate the parameter } \\
\hline Step & $\begin{array}{l}\text { Serial number of } \\
\text { experiments implied }\end{array}$ & Type & $\sigma_{f} / \sigma_{y}$ & Step & $\begin{array}{l}\text { Serial number of } \\
\text { experiments implied }\end{array}$ & Type & $\sigma_{v} / \sigma_{y}$ \\
\hline NA & 0 & Table 1 & NA & NA & $4-12$ & Constant & 23 \\
\hline \#1 & 1 & Dynamic & 1.09 & NA & $4-12$ & Constant & 2.60 \\
\hline$\# 2$ & 1,2 & Dynamic & 1.01 & NA & $4-12$ & Constant & 2.31 \\
\hline \#3 & $1-3$ & Dynamic & 1.34 & \#4 & $4-12$ & Constant & 4.33 \\
\hline \#5 & $4-12$ & Constant & 1.73 & \#6 & $1-3$ & Dynamic & 3.25 \\
\hline
\end{tabular}

21 randomly picked initial guesses were found to be sufficient to obtain an acceptable criterion on protocol optimization. Practically, it was found that increasing the number of initial guesses did not improve the overall performance of the protocol optimization (optimality criterion).

We checked that 10 initial guesses were enough to obtain an acceptable estimation of parameter vector, by observing that all minimizations either converged to the same neighbourhood or exited with high residuals.
A variation of Table 2, not shown in this paper, was generated, taking an initial guess for the parameters which is far from the solution (namely $\left.\theta^{(0)}=\left[10^{6} \mu \mathrm{m}^{2} / \mathrm{s}, 10^{5} \mu \mathrm{m}^{2} / \mathrm{s}, 150 \mathrm{~W} / \mathrm{Km}^{2}, 170 \mathrm{~W} / \mathrm{Km}^{2}, 0.001\right]\right)$. It was verified that the optimally designed estimations still converged to the solution: estimation based only on first optimally designed experiment gave a parameter which is quite close to the value of the "true parameter" shown in Table 2(d), for A- and D-optimality.
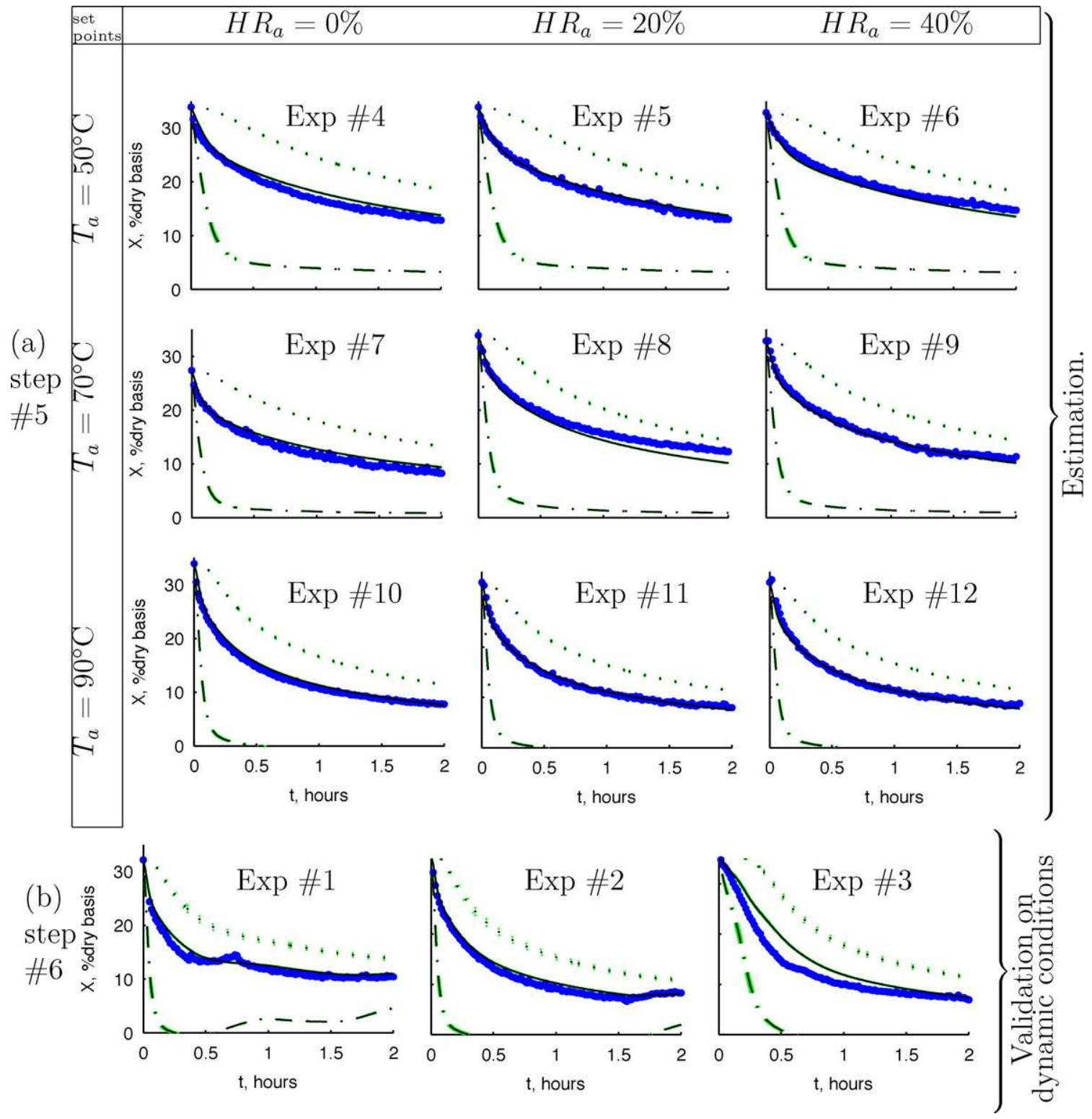

Fig. 7. (a) Nine constant-condition drying experiment and (b) the validation step. Mean moisture content predicted by model for inner compartment (..), outer compartment $(-\cdot)$; mean moisture content recorded (. in dark blue) and predicted by model (- in black). Confidence bands of predictions are in light green. All simulations are based only on parameters computed from data recorded from experiments Exp. \#4-12 shown in (a). (For interpretation of the references to color in this figure legend, the reader is referred to the web version of this article.) 


\section{Discussion}

A-optimality performed better than D-optimality in our case; this is also the case of other works [40, 3, p. 395, 397]. The big differences between the marginal confidence intervals obtained with the different design criteria (Table 2 ) is probably due to the strong nonlinearities in the model

The A-optimally designed experiment depends on the choice of the reparameterization of the parameters: hence the prerequisites (2) and (3) in Section 2.3 are important to ensure that each parameter is identified with a confidence interval consistent with its own relative influence on the predictions.

The computational cost $(290,000$ model calls), compatible with the cost of global optimizers, is due to a naive implementation of assumption (f2) defined in Section 3.4.2. This assumption is a constraint on the optimizer to avoid the condensation forbidden at end of Section 3.3. The naive implementation alternates up to 20 times the optimization of the design criterion with the optimization of the duration of experiment without condensation. We think a better implementation of assumption (f2) would result in a gain of at least an order of magnitude.

The influence of unmatched increase of water content of Exp. \#1 on the prediction seems to decrease when more experiences are taken in account. Ideally, this unmatched could have been eliminated from the data, but we were not able to find an objective criterion to eliminate that data. It would be possible if condensation was assessed by independent measures that are not available on the pilot.

As the final confidence ellipsoid will, in this article, not be close to the boundaries, the loss of information of DoEL methods are of the order of magnitude of $14 \%$ when compared to DoENL methods [1, p. 1144].

\section{Conclusions}

We used a methodology which lets us replace nine experiments with static dry ing conditions by three A-optimally designed experiments with dynamic conditions. Our methodology let us divide by three the time of experimentation and the amount of used product. The confidence intervals on the identified parameters were quite as effective.

Our methodology of sequential DoEL was tested in a virtual environment; the A-optimality was shown to be somehow better than the E-optimality and far better than the D-optimality. It was observed that first experiment reaches directly the right parameter neighborhood in terms of order of magnitude.

We used reparameterization of the parameter and protocol vectors which let us use standard local optimisation algorithms to make design of experiment (DoE) on models without analytical solution and the use of global optimizer or proprietary optimizers was avoided (such optimizers are used in existing works doing DoEs on model without analytical solution $[7,8,41,42]$, and the typical global optimizer calls $10^{6}$ times the model subroutine [9]). We used standard local optimizers for DoE of a nonlinear model that have been confronted to real experiments.

We used degraded DoEL methods: in the experimental environment, experimental temperature profile might differ, to some extent, from the designed one; we used criteria of design (A-, D- and E-optimality) that are less exact than X-optimality for models nonlinear in $\theta$. This methodology was shown to give nonetheless good results: identifying simultaneously five transfer-related constants in three Aoptimally designed experiments gave a precision only $33 \%$ worse than the classical identification based on nine experiments with static drying conditions. Hence, this methodology let us divide by three the time of experimentation, and the amount of used product, for a similar quality of identification.

It is generally observed that the computed A-optimal protocol tends to maximize the amplitude of the variations of experimental conditions. The proposed methodology has shown, on this particular example, its ability to minimize the experimental work needed to identify unknowns in a given non-linear dynamic model. Furthermore, it optimizes confidence levels on the estimates while classical strategies cannot give any guarantee on that point.

Current work in progress is trying to apply this so-called generic method to totally different classes of dynamic models. Supplementary research and simulations are planned to study the sensibility of the optimal design to the choice of the various constants, the optimality criteria and the minimization algorithms. This would constitute a useful guide for the drying experimentator.

\section{Acknowledgements}

This work has been partly funded by Agence Nationale de la Recherche within Project ANR-06-PNRA-023 REACTIAL "Prediction and control of the appearance or disappearance of reactional markers during food process and conservation".

\section{Appendix A. Model of pilot inertias}

The combination $\left(H_{R a \infty}-H_{R a}\right) / H_{R a 5}-\left(T_{a \infty}-T_{a}\right) / T_{a 5}$ has an exponential decrease $\exp \left(-t / t_{5}\right)$; the combination $\left(H_{R a \infty}-H_{R a}\right) / H_{R a 6}+\left(T_{a \infty}-T_{a}\right) / T_{a 6}$ has a Gompertz decrease $\exp \left(\left(1-e^{-t / t_{4}}\right) / t_{3}\right)$ (which corresponds to the Gompertz adimensional constant $\left.c=\exp \left(t_{4} / t_{3}\right)\right)$.
Expanding these details, we obtain the following non-linear model for air temperature and relative humidity set point changes, based on air temperature $T_{a 0}$ and relative humidity $H_{R a 0}$ before set point change, and at set point air temperature $T_{a \infty}$ and relative humidity $H_{R a \infty}$ :

$$
\begin{aligned}
& T_{a}=T_{a 0}+T_{a 3} \times\left(1-e^{e^{-\left(t_{3} / t_{4}\right)}-e^{\left.\left(t-t_{3}\right) / t_{4}\right)}}\right) \times\left(\frac{H_{R a \infty}-H_{R a 0}}{H_{R a 6}}+\frac{T_{a \infty}-T_{a 0}}{T_{a 6}}\right) \\
& -T_{a 4} \times\left(1-e^{-\left(t / t_{5}\right)}\right) \times\left(\frac{H_{R a \infty}-H_{R a 0}}{H_{R a 5}}-\frac{T_{a \infty}-T_{a 0}}{T_{a 5}}\right) \\
& H_{R a}=H_{R a 0}+H_{R a 3} \times\left(1-e^{e^{-\left(t_{3} / t_{4}\right)}-e^{\left(t-t_{3}\right) / t_{4}}}\right) \times\left(\frac{H_{R a \infty}-H_{R a 0}}{H_{R a 6}}+\frac{T_{a \infty}-T_{a 0}}{T_{a 6}}\right) \\
& +H_{R a 4} \times\left(1-e^{-\left(t / t_{5}\right)}\right) \times\left(\frac{H_{R a \infty}-H_{R a 0}}{H_{R a 5}}-\frac{T_{a \infty}-T_{a 0}}{T_{a 5}}\right)
\end{aligned}
$$

The fit between this model Eqs. (A.1) and (A.2) and dedicated $15 \mathrm{~h}$ of experiments is shown in Fig. 4.

\section{References}

[1] J.P. Gauchi, A. Pázman, Designs in nonlinear regression by stochastic minimization of functionals of the mean square error matrix, J. Stat. Plan. Inference 136 (2006) $1135-1152$.

[2] J.-P. Vila, J.-P. Gauchi, Optimal designs based on exact confidence regions for parameter estimation of a nonlinear regression model, J. Stat. Plan. Inference 137 (2007) 2935-2953.

[3] A. Pázman, L. Pronzato, Nonlinear experimental design based on the distribution of estimators, J. Stat. Plan. Inference 33 (1992) 385-402.

[4] G. Franceschini, S. Macchietto, Model-based design of experiments for parameter precision: State of the art, Chem. Eng. Sci. 63 (2008) 4846-4872.

[5] D. Ucinski, Optimal Measurement Methods for Distributed Parameter System Identification, CRC Press, 2005.

[6] F.P. Casey, D. Baird, Q. Feng, R.N. Gutenkunst, J.J. Waterfall, C.R. Myers, K.S. Brown, R.A. Cerione, J.P. Sethna, Optimal experimental design in an epidermal growth factor receptor signalling and down-regulation model, IET Syst. Biol. 1 (2007) 190-202.

[7] M. Rodriguez-Fernandez, P. Mendes, J.R. Banga, A hybrid approach for efficient and robust parameter estimation in biochemical pathways, Biol. Syst. 83 (2006) $248-265$.

[8] E. Balsa-Canto, M. Rodriguez-Fernandez, J.R. Banga, Optimal design of dynamic experiments for improved estimation of kinetic parameters of thermal degradation, J. Food Eng. 82 (2007) 178-188.

[9] C.G. Moles, P. Mendes, J.R. Banga, Parameter estimation in biochemical pathways: a comparison of global optimization methods, Genome Res. 13 (2003) 2467-2474.

[10] F. Courtois, M. Abud Archila, C. Bonazzi, J.M. Meot, G. Trystram, Modeling and control of a mixed-flow rice dryer with emphasis on breakage quality, J. Food Eng. 49 (2001) 303-309.

[11] M.A.J.S. van Boekel, Kinetic Modeling of Reactions In Foods, CRC Press, 2008.

[12] A.K. Agarwal, M.L. Brisk, Sequential experimental design for precise parameter estimation. 1. Use of reparameterization. Ind. Eng. Chem. Process Des. Dev. 24 (1985) 203-207.

[13] V.G. Dovi, Optimal experiment planning for environmental kinetic models subject to data heteroscedasticity, Environmetrics 8 (1997) 303-311.

[14] V.G. Dovi, A.P. Reverberi, L. Acevedo-Duarte, New procedure for optimal design of sequential experiments in kinetic models, Ind. Eng. Chem. Res. 33 (1994) 62-68.

[15] D.M. Espie, S. Macchietto, Nonlinear transformations for parameter estimation, Ind. Eng. Chem. Res. 27 (1988) 2175-2179.

[16] K. Worden, Data processing and experiment design for the restoring force surface method, part ii: choice of excitation signal, Mech. Syst. Signal Process. 4 (1990) 321-344.

[17] S. Phoungchandang, S. Nongsang, P. Sanchai, The development of ginger drying using tray drying, heat pump-dehumidified drying, and mixed-mode solar drying, Dry. Technol. 27 (2009) 1123-1131.

[18] A. Tarmian, A. Sepeher, S. Rahimi, Drying stress and strain in tension wood: a conventional kiln schedule to efficiently dry mixed tension/normal wood boards in poplar, Dry. Technol. 27 (2009) 1033.

[19] W.T. Simpson (Ed.), Dry Kiln Operator's Manual, vol. 188, Madison, Wisconsin, 2001.

[20] A.C. Atkinson, B. Bogacka, Compound and other optimum designs for systems of nonlinear differential equations arising in chemical kinetics, Chemom. Intell. Lab. Syst. 61 (2002) 17-33.

[21] H.B. Pfost, S.G. Maurer, D.S. Chung, G.A. Milliken, Summarizing and reporting equilibrium moisture data for grains, Trans. ASAE 3520 (1976).

[22] M. Abud-Archila, F. Courtois, C. Bonazzi, J.J. Bimbenet, Processing quality of rough rice during drying - modelling of head rice yield versus moisture gradients and kernel temperature, J. Food Eng. 45 (2000) 161-169.

[23] S. Sokhansanj, W. Yang, Revision of the ASAE standard d245.4: moisture relationships of grains, Trans. ASAE 39 (1996) 639-642.

[24] E. Walter, L. Pronzato, Identification of Parametric Models from Experimental Data, Springer, London, 2010 
[25] A.E. Bryson, Y.-C. Ho, Applied Optimal Control: Optimization, Estimation, and Control, Taylor \& Francis, New York, 1975

[26] K.J. Versyck, K. Bernaerts, A.H. Geeraerd, J.F. Van Impe, Introducing optimal experimental design in predictive modeling: a motivating example, Int. J. Food Microbiol. 51 (1999) 39-51.

[27] D.B. Leineweber, I. Bauer, H.G. Bock, J.P. Schlöder, An efficient multiple shooting based reduced SQP strategy for large-scale dynamic process optimization. Part 1: theoretical aspects, Comput. Chem. Eng. 27 (2003) 157-166.

[28] A. Cihan, M.C. Ece, Liquid diffusion model for intermittent drying of rough rice, J. Food Eng. 49 (2001) 327-331.

[29] R. Lu, T.J. Siebenmorgen, T.R.G. Archer, Absorption of water in long-grain rough rice during soaking, J. Food Process Eng. 17 (1994) 141-154.

[30] J.F. Steffe, R.P. Singh, Diffusion coefficients for predicting rice drying behaviour, J. Agric. Eng. Res. 27 (1982) 489-493.

[31] J.-J. Bimbenet, A. Duquenoy, G. Trystram, Génie des procédés alimentaires: Des bases aux applications, Dunod - RIA, 2002

[32] K. Toyoda, Study on intermittent drying of rough rice in a recirculation dryer, in: M.A. Roques, A.S. Mujumdar (Eds.), Sixth International Drying Symposium IDS'88, Kobe Univ., Kobe, Japan, 1988, pp. 171-178.

[33] H.G. Bock, Numerical treatment of inverse problems in chemical reaction kinetics, in: K.H. Ebert, P. Deuffhard, W. Jager (Eds.), Modelling of Chemical Reaction Systems: Proceedings of an International Workshop, vol. 18, Springer-Verlag, 1981, pp. 102-125.

[34] J.R. Leis, M.A. Kramer, Algorithm 658: Odessa-an ordinary differential equation solver with explicit simultaneous sensitivity analysis, ACM Trans. Math. Softw. 14 (1988) 61-67
[35] N.R. Draper, H. Smith, Applied Regression Analysis, Wiley Series in Probability and Statistics. Texts and References Section, John Wiley \& Sons Inc, New York, 1998.

[36] E. Kreyszig Introductory Mathematical Statistics, John Wiley \& Sons Inc, 1970.

[37] Afnor (Ed.), Céréales et produits céréaliers, Recueil de normes francaises, 1982.

[38] J.C. Lagarias, J.A. Reeds, M.H. Wright, P.E. Wright, Convergence properties of the Nelder-Mead simplex method in low dimensions, SIAM J. Optim. 9 (1998) 112-147.

[39] J. Moré, The Levenberg-Marquardt algorithm: implementation and theory, in: G. Watson (Ed.), Numerical Analysis, Vol. 630 of Lecture Notes in Mathematics, Springer, Berlin/Heidelberg, 1978, pp. 105-116.

[40] J. Mulder, W. van der Linden, Multidimensional adaptive testing with optimal design criteria for item selection, Psychometrika 74 (2009) 273-296.

[41] S.B. Shin, S.P. Han, W.J. Lee, Y.H. Im, J.H. Chae, D.-i. Lee, W.H. Lee, Z.G.E. Urban, Optimize terephthaldehyde reactor operations, Hydrocarb. Process. 86 (2007) 83-90.

[42] C. Bäumler, M. Matzopoulos, Z.G.E. Urban, Enhanced methods optimize ownership costs for catalysts, Hydrocarb. Process. 86 (2007) 71-78.

[43] T.H. Chilton, A.P. Colburn, Mass transfer (absorption) coefficients, Ind. Eng. Chem. 26 (1934) 1183-1187.

[44] R.P. Singh, D.R. Heldman, Introduction to Food Engineering, Academic Press Inc, 2008

[45] M. Abud Archila, JJ. Bimbenet, Modélisation simultanée des transferts et de l'évolution de la qualité technologique du riz paddy en vue d'optimiser les conditions de séchage, Ph.D. thesis, Ecole nationale supérieure des industries agricoles et alimentaires, Massy, France, 2000. 